\title{
TOXICIDADE AGUDA E ANÁLISE DE RISCO DE HERBICIDAS E INSETICIDAS UTILIZADOS NA LAVOURA DO ARROZ IRRIGADO SOBRE O CLADÓCERO Daphnia magna
}

\author{
FERNANDO KOJIMA NAKAGOME* \\ JOSÉ ALBERTO NOLDIN** \\ CHARRID RESGALLA JR. ***
}

\begin{abstract}
Este trabalho teve como objetivo determinar a $\mathrm{CE}_{50 ; 48 \mathrm{~h}}$ de alguns herbicidas e inseticidas utilizados na cultura do arroz irrigado e estimar os coeficientes de risco (análise de risco determinístico), com base na toxicidade dos compostos para Daphnia magna. Foram avaliados os herbicidas oxyfluorfem, oxadiazona, carbofentrazona etílica, clomazona, quincloraque, pirazossulfurom etílico, 2,4-D, bispiribaque-sódico, metsulfurom metílico e bentazona e os inseticidas lambdacialotrina, fipronil e carbofurano. Os resultados indicaram que os herbicidas oxyfluorfem, oxadiazona e os inseticidas lambdacialotrina, fipronil e carbofurano são os produtos com maior toxicidade sobre $D$. magna. Os valores de $\mathrm{CE}_{50} ; 48 \mathrm{~h}$ em mg. $\mathrm{L}^{-1}$ obtidos foram: 3,$18 ; 3,54 ; 12,252 ; 14,35 ; 134,31$; 182,$11 ; 407,3 ; 1.060,0 ; 2.100,0 ; 3.900,0 ; 0,00021$; 0,15 e 0,32 , respectivamente, para os ingredientes ativos oxyfluorfem, oxadiazona, carbofentrazona etílica, clomazona, quincloraque, pirazossulrurom etílico, 2,4-D, bispiribaque-sódico, metsulfurom metílico, bentazona, lambdacialotrina, fipronil e carbofurano. Considerando a dose de aplicação recomendada pelo fabricante diluída em lâmina de água de $10 \mathrm{~cm}$ na cultura do arroz irrigado, os erbicidas oxyfluorfem, oxadiazona e os inseticidas lambdacialotrina, fipronil e carbofurano merecem cuidados no manejo da água de irrigação da lavoura.
\end{abstract}

PALAVRAS-CHAVE: OXYFLUORFEM; OXADIAZONA; CARBOFENTRAZONA ETÍLICA; CLOMAZONA; QUINCLORAQUE; PIRAZOSSULFURON-ETÍLICO; 2,4-D; BISPIRIBAQUE-SÓDICO; METSULFUROM METÍLICO; BENTAZONA; LAMBDACIALOTRINA; FIPRONIL; CARBOFURANO; ECOTOXICOLOGIA.

Oceanógrafo, CTTMar, Universidade do Vale do Itajaí (UNIVALI), Itajaí, SC (e-mail: fernando nakagome@gmail.com).

* $\quad$ Ph.D. em Agronomia, Pesquisador EPAGRI/ Estação Experimental de Itajaí, SC (e-mail; noldi@epagri.rctsc.br).

*** Doutor em Fisiologia Geral, Professor do CTTMar/UNIVALI, Itajaí, SC (e-mail: cresgalla@univali.br). 


\section{INTRODUÇÃO}

O arroz irrigado, cultura de destaque no sul do Brasil, ocupa área de aproximadamente 1,3 milhão hectares nos estados do Rio Grande do Sul e Santa Catarina, que fornecem quase $60 \%$ da produção nacional (CONAB, 2006).

Santa Catarina, com área de aproximadamente 145 mil hectares cultivada com arroz irrigado, destaca-se como o primeiro estado em produtividade e o segundo em volume de produção (CONAB, 2006). O sistema de cultivo em Santa Catarina caracteriza-se pela utilização de sementes prégerminadas, semeadas a lanço em solo coberto por lâmina de água (EPAGRI, 2005).

A obtenção de alta produtividade em arroz irrigado, e também no sistema pré-germinado, está diretamente relacionada com o manejo adequado dos principais fatores que causam danos à cultura, especialmente plantas daninhas, pragas, doenças e nutrição das plantas. Para o manejo desses limitantes são utilizados diversos agroquímicos, incluindo herbicidas, inseticidas, fungicidas e adubos. No sistema pré-germinado, geralmente os insumos químicos são aplicados antecedendo a semeadura (adubos), via tratamento das sementes (inseticidas) ou em pós-emergência (herbicidas, inseticidas e adubos), diretamente na lâmina de água ou a lavoura é inundada após a aplicação, especialmente de herbicidas.

Segundo a SOSBAI (2005) são recomendados, no Brasil, para a cultura do arroz irrigado 23 ingredientes ativos de herbicidas (comercializados isoladamente ou em mistura na forma de 37 produtos comerciais), 14 ingredientes ativos de inseticidas ( 25 produtos comerciais) e 25 ingredientes ativos de fungicidas, (40 produtos comerciais).

Existe carência de informações sobre os possíveis impactos que os agrotóxicos podem causar em sistemas aquáticos, porém sabe-se dos riscos de transporte desses das lavouras para as águas superficiais, podendo resultar numa ação direta sobre organismos não-alvos ou indireta pela alteração do seu habitat ou interferência na cadeia alimentar.

Os peixes e animais aquáticos são expostos aos pesticidas, segundo HELFRICH et al. (1996), mediante ingestão de alimento contaminado, através da pele ao nadar em águas contaminadas e por meio da respiração, absorvendo-os pelas brânquias até atingir equilíbrio, dependendo das condições físico-quimicas e da concentração do composto (SEUS, 2002).

As espécies do gênero Daphnia, conhecidas popularmente como "pulgas d'água", constituem importante fonte de alimentos para os peixes e são freqüentemente utilizadas como bioindicadores em ensaios ecotoxicológicos. Na França, Daphnia magna foi uma das espécies escolhidas para o monitoramento de efluentes industriais e agentes tóxicos. Nos Estados Unidos da América, o uso dessa espécie é recomendado pela Environmental Protection Agency (EPA) para a avaliação da toxicidade de agroquímicos (ZAGATTO e GOLDSTEIN, 1984).

Diversos estudos já foram realizados para avaliar a toxicidade de agroquímicos, destacandose o trabalho de HELFRICH et al. (1996) que utilizaram a truta arco-íris (Oncorhynchus mykiss) para estimar a toxicidade do herbicida oxyfluorfem (Goal BR) e do inseticida carbofurano (Furadan). FRELLO (1998) utilizou Daphnia magna e Poecilia reticulata para avaliar a toxicidade do carboforano. JONSSON e MAIA (1998) estimaram a CL ${ }_{50}$; $96 \mathrm{~h}$ para o peixe Hyphessobrycon scholzei para diversos herbicidas. No trabalho realizado por RESGALLA JÚNIOR et al. (2002) foram estimados índices de segurança para diferentes produtos, utilizando a carpa-comum (Cyprinus carpio) como organismo-teste.

O objetivo deste trabalho foi determinar a concentração efetiva mediana $\left(\mathrm{CE}_{50}, 48 \mathrm{~h}\right)$ de alguns agroquímicos, utilizados na cultura de arroz irrigado e estimar os seus coeficientes de risco (análise de risco determinístico) com base na toxicidade dos produtos para o cladócera Daphnia magna.

\section{MATERIAL E MÉTODOS}

Os testes de toxicidade foram realizados conforme a NBR 12713 da ABNT (2004), sendo 
conduzidos no Laboratório de Ecotoxicologia da Universidade do Vale do Itajaí (UNIVALI). Utilizaramse nos testes os herbicidas 2,4-D (U46 D-Fluid 2,4-D - 720 g i.a. L-1), metsulfurom metílico (Ally - 600 g i.a. $\mathrm{kg}^{-1}$ ), carbofentrazona etílica (Aurora $400 \mathrm{CE}-400 \mathrm{~g}$ i.a. $\mathrm{L}^{-1}$ ), bentazona (Basagran $600-600 \mathrm{~g}$ i.a. $\mathrm{L}^{-1}$ ), quincloraque (Facet PM $-500 \mathrm{~g}$ i.a. $\mathrm{kg}^{-1}$ ), clomazona (Gamit $-500 \mathrm{~g}$ i.a. $\mathrm{L}^{-1}$ ), oxifluorfem (Goal BR - 240 g i.a. L-1), bispiribaque-sódico (Nominee 400 SC - 400 g i.a. L-1), oxadiazona (Ronstar 250 BR -250 g i.a. $L^{-1}$ ), pirazossulrurom etílico (Sírius -250 g i.a. $L^{-1}$ ), e os inseticidas carbofurano (Furadan $50 \mathrm{G}-50 \mathrm{~g}$ i.a. $\mathrm{kg}^{-1}$ ), lambdacialotrina (Karate $50 \mathrm{EC}-50 \mathrm{~g}$ i.a. $\mathrm{L}^{-1}$ ) e fipronil (Standak $250 \mathrm{FS}-250$ g i.a. $\left.\mathrm{L}^{-1}\right)$, além de dicromato de potássio $\left(\mathrm{K}_{2} \mathrm{Cr}_{2} \mathrm{O}_{7}\right)$ como substância de referência para o teste de sensibilidade da espécie utilizada.

Juvenis de Daphnia magna foram obtidos em cultivo no laboratório com idade variando de 2 a 26 horas. Seis concentrações de cada agroquímico foram selecionadas a partir de testes preliminares. Para cada teste foram utilizados frascos controle para validação do experimento, que constou na manutenção dos organismos em meio de cultura. Os frascos-teste (béqueres de $50 \mathrm{~mL}$ ) em duplicata continham 20 organismos e $40 \mathrm{~mL}$ da solução-teste, sendo mantidos em incubadoras com temperatura de $20 \pm 2^{\circ} \mathrm{C}$ e fotoperíodo de 12 horas de luz. Os testes duraram 48 horas.

Ao final dos testes registrou-se o número de organismos imóveis em cada frasco. Os indivíduos foram considerados imóveis quando não apresentavam movimento após 10 segundos de observação mesmo com pequena agitação dos béqueres.

Os dados foram analisados segundo recomendações da USEPA (2002), utilizando-se o programa TSK ( Trimmed Spearman-Karber) para a obtenção da $\mathrm{CE}_{50}$; 48h (concentração efetiva mediana de 48 horas).

Após a determinação da $\mathrm{CE}_{50} ; 48 \mathrm{~h}$, os agroquímicos foram classificados segundo o critério proposto por HELFRICH et al. (1996) que estabelece a toxicidade dos produtos com base nos valores de $C E_{50}$, em mg.L-1: mínima $=>100 ;$ leve $=11$ a 100; moderada $=1,1$, a 10; alta =0,11 a 1,0, extrema $=0,01$ a 1 , e super-extrema $=<0,01$.

Comparou-se a $\mathrm{CE}_{50} ; 48 \mathrm{~h}$ dos compostos analisados com suas respectivas concentrações estimadas no ambiente para a obtenção dos coeficientes de risco determísticos. Para isto, considerou-se a dose recomendada pelo fabricante (Tabela 1) e a diluição em lâmina d'água de $10 \mathrm{~cm}$. Os coeficientes de risco estimados foram: índice de segurança de SOLOMON (1997), que é a razão entre a CE $\mathrm{E}_{50}$ sobre a concentração estimada (valores resultantes superiores a 20 indicam ausência de risco ecológico); índice de risco da USEPA (1999), que é a razão entre a concentração estimada sobre a $\mathrm{CE}_{50}$ (valores resultantes inferiores a 0,1 indicam ausência de risco ecológico) e índice de KOKTA e ROTHERT (1992), que é a comparação entre os valores de $\mathrm{CE}_{50}$ com a concentração estimada multiplicada por 10, (se o valor de $\mathrm{CE}_{50}$ for superior não existe risco ecológico).

\section{RESULTADOS E DISCUSSÃO}

\subsection{TESTE DE SENSIBILIDADE}

Foram realizados cinco testes de toxicidade com $D$. magna sob efeito da substância de referência dicromato de potássio, intercaladamente, entre os experimentos com os agroquímicos. $O$ valor médio da $\mathrm{CE}_{50}$; $48 \mathrm{~h}$ obtido foi de $0,51 \mathrm{mg} \cdot \mathrm{L}^{-1}$ com desvio-padrão de 0,12 e coeficiente de variação (CV) de $24,5 \%$. Esse resultado enquadra-se no limite de variabilidade de $45 \%$ do CV proposto pela USEPA (2002).

\subsection{VALORES DE CE 50 ; 48h E ANÁLISE DE RISCO PARA OS AGROQUÍMICOS}

Os valores de $\mathrm{CE}_{50} ; 48 \mathrm{~h}$ dos produtos e ingredientes ativos utilizados nos testes com o cladócera D.magna são apresentados na Quadro 1. 
Os herbicidas apresentaram baixa toxicidade sobre $D$. magna e enquadraram-se na classificação de toxicidade mínima pelo critério HELFRICH et al. (1996), com exceção dos herbicidas oxadiazona e oxyfluorfem (toxicidade moderada). Todos os inseticidas avaliados enquadraram-se na classificação de periculosidade alta, tendo o produto Karate apresentado toxicidade super-extrema para $D$. magna. Entretanto, essa classificação é limitada por não considerar as concentrações de aplicação. Desta forma, os índices de segurança ou índices de risco determinístico podem revelar a toxicidade real dos agroquímicos confirmando ou não a classificação anterior.

\section{QUADRO 1 - AGROQUÍMICOS, VALORES DE CE $_{50} ; 48$ HORAS PARA OS PRODUTOS COMERCIAIS E INGREDIENTES ATIVOS, INTERVALOS DE CONFIANÇA E TOXICIDADE SEGUNDO A CLASSIFICAÇÃO DE HELFRICH et al. (1996)}

\begin{tabular}{|c|c|c|c|c|c|c|}
\hline & $\begin{array}{l}\text { Produto } \\
\text { comercial }\end{array}$ & Nome comum & $\mathrm{CE}_{50}$ (p.c.) & $\begin{array}{c}\mathrm{CE}_{50} \text { (i.a.) } \\
\left(\mathrm{mg} \cdot \mathrm{L}^{-1}\right)\end{array}$ & $\begin{array}{l}\text { Intervalo de } \\
\text { confiança } \\
\text { (IC 95\%) (i.a.) }\end{array}$ & Toxicidade \\
\hline \multirow{10}{*}{$\begin{array}{l}\frac{9}{8} \\
\frac{\pi}{0} \\
: \frac{0}{0} \\
\frac{0}{0} \\
\frac{10}{1} \\
\end{array}$} & $\begin{array}{l}\text { U46 D- } \\
\text { Fluid }\end{array}$ & $2,4-D$ & $565,69 \mu \mathrm{L} . \mathrm{L}^{-1}$ & 407,30 & - & mínima \\
\hline & Ally & $\begin{array}{l}\text { metsulfurom- } \\
\text { metílico }\end{array}$ & $3500 \mathrm{mg} \cdot \mathrm{L}^{-1}$ & 2100 & $\pm 0,20$ & mínima \\
\hline & Aurora & $\begin{array}{c}\text { carbofentrazona } \\
\text { etílica }\end{array}$ & $30,63 \mu \mathrm{L} \cdot \mathrm{L}^{-1}$ & 12,25 & $\pm 1,65$ & leve \\
\hline & Gamit & clomazona & $28,70 \mu \mathrm{L} . \mathrm{L}^{-1}$ & 14,35 & $\pm 1,59$ & leve \\
\hline & Goal & oxyfluorfem & $13,13 \mu \mathrm{L} . \mathrm{L}^{-1}$ & 3,18 & $\pm 0,82$ & moderada \\
\hline & Nominee & $\begin{array}{l}\text { bispiribaque } \\
\text { sódico }\end{array}$ & $2,63 \mathrm{~mL} \cdot \mathrm{L}^{-1}$ & 1060 & $\pm 0,08$ & mínima \\
\hline & Ronstar & oxadiazona & $14,14 \mu \mathrm{L} . \mathrm{L}^{-1}$ & 3,54 & $\pm 0,57$ & moderada \\
\hline & Facet & quincloraque & $268,63 \mathrm{mg} \cdot \mathrm{L}^{-1}$ & 134,31 & $\pm 7,90$ & mínima \\
\hline & Sírius & $\begin{array}{c}\text { pirazossulfurom } \\
\text { etílico }\end{array}$ & $0,73 \mathrm{~mL} \cdot \mathrm{L}^{-1}$ & 182,11 & $\pm 8,12$ & mínima \\
\hline & Basagran & bentazona & $6,50 \mathrm{~mL} \cdot \mathrm{L}^{-1}$ & 3900 & $\pm 0,42$ & mínima \\
\hline \multirow{3}{*}{ 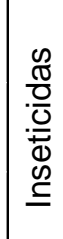 } & Standak & fipronil & $0,61 \mu \mathrm{L} \cdot \mathrm{L}^{-1}$ & 0,15 & $\pm 0,10$ & alta \\
\hline & Furadan & carbofurano & $6,37 \mathrm{mg} \cdot \mathrm{L}^{-1}$ & 0,32 & $\pm 0,03$ & alta \\
\hline & Karate & Lambdacialotrina & $4,20 \mu \mathrm{L} \cdot \mathrm{L}^{-1}$ & 0,00021 & $\pm 0,03$ & super-extrema \\
\hline
\end{tabular}

No Quadro 2 são apresentados, em ordem decrescente de risco ecológico, os produtos testados. Segundo esses resultados, a periculosidade e o risco ecológico foram confirmados para os produtos lambdacialotrina, carbofurano, oxadiazona, fipronil e oxyfluorfem. Essa classificação alerta para os cuidados a serem tomados no manuseio, estocagem e, principalmente, no manejo da água após a aplicação dos produtos na cultura do arroz irrigado. Resultados similares foram obtidos por RESGALLA JÚNIOR et al. (2002) com base na letalidade do peixe Cyprinus carpio.

A diferenciação na toxicidade sobre $D$. magna entre herbicidas e inseticidas deve-se ao modo de ação do agroquímico. Enquanto os herbicidas exercem ação específica sobre vegetais, os inseticidas 
atuam no controle de animais (insetos). Os herbicidas agem normalmente em biomoléculas específicas de vegetais (como o ácido indol-3-acético, auxina principal de várias plantas) interferindo na expansão, alongamento e divisão celular (RODRIGUES e ALMEIDA, 2005). Diferentemente, os inseticidas atuam de forma neurológica.

\section{QUADRO 2 - VALORES DE CE $_{50} ; 48$ HORAS PARA Daphinia magna E CONCENTRAÇÃO ESTIMADA PARA OS INGREDIENTES ATIVOS E OS COEFICIENTES DE RISCO DETERMINÍSTICOS}

\begin{tabular}{|c|c|c|c|c|c|}
\hline Produtos & $\mathrm{CE}_{50}, 48 \mathrm{~h}$ & $\begin{array}{l}\text { Concentraçãa } \\
\text { estimada' }\end{array}$ & $\begin{array}{c}\text { Índice de } \\
\text { segurança } \\
\text { (SOLOMON,1997) }\end{array}$ & $\begin{array}{l}\text { Coeficiente de } \\
\text { risco } \\
\text { (USEPA, 1999) }\end{array}$ & $\begin{array}{c}\text { Concentração estimada } \\
\text { x } 10 \text { (KOKTAe } \\
\text { ROTHERT,1992) }\end{array}$ \\
\hline Karatê & 0,21 ug. $L^{-1}$ & $50 \mu g \cdot L^{-1}$ & $0,0042^{*}$ & $238,095^{*}$ & $\mathrm{CE}_{50}<500 \mu \mathrm{g} \cdot \mathrm{L}^{-1} *$ \\
\hline Furadan & $0,32 \mathrm{mg} \cdot \mathrm{L}^{-1}$ & $1 \mathrm{mg} \cdot \mathrm{L}^{-1}$ & $0,32 *$ & $3,125^{*}$ & $\mathrm{CE}_{50}<10 \mathrm{mg} \cdot \mathrm{L}^{-1}$ * \\
\hline Ronstar & $3,54 \mathrm{mg} \cdot \mathrm{L}^{-1}$ & $1 \mathrm{mg} \cdot \mathrm{L}^{-1}$ & $3,54^{*}$ & $0,282 *$ & $C E_{50}<10 \mathrm{mg} \cdot \mathrm{L}^{-1}$ * \\
\hline Standak & $0,15 \mathrm{mg} \cdot \mathrm{L}^{-1}$ & $40 \mu \mathrm{g} \cdot \mathrm{L}^{-1}$ & $3,75^{*}$ & $0,267^{*}$ & $C E_{50}<0,4 \mathrm{mg} \cdot \mathrm{L}^{-1}$ * \\
\hline Goal & $3,18 \mathrm{mg} \cdot \mathrm{L}^{-1}$ & $0,36 \mathrm{mg} \cdot \mathrm{L}^{-1}$ & $8,83 *$ & $0,113^{*}$ & $\mathrm{CE}_{50}<3,6 \mathrm{mg} \cdot \mathrm{L}^{-1}$ * \\
\hline Gamit & $14,35 \mathrm{mg} \cdot \mathrm{L}^{-1}$ & $0,7 \mathrm{mg} \cdot \mathrm{L}^{-1}$ & 20,5 & 0,049 & $\mathrm{CE}_{50}>7 \mathrm{mg} \cdot \mathrm{L}^{-1}$ \\
\hline Aurora & $12,25 \mathrm{mg} \cdot \mathrm{L}^{-1}$ & $60 \mu \mathrm{g} \cdot \mathrm{L}^{-1}$ & 204,2 & 0,004897 & $\mathrm{CE}_{50}>600 \mu \mathrm{g} \cdot \mathrm{L}^{-1}$ \\
\hline Facet & $134,31 \mathrm{mg} \cdot \mathrm{L}^{-1}$ & $0,375 \mathrm{mg} \cdot \mathrm{L}^{-1}$ & 358,2 & 0,00279 & $\mathrm{CE}_{50}>3,75 \mathrm{mg} \cdot \mathrm{L}^{-1}$ \\
\hline $2,4-D$ & $407,30 \mathrm{mg} \mathrm{L}^{-1}$ & $0,72 \mathrm{mg} \cdot \mathrm{L}^{-1}$ & 565,7 & 0,00177 & $\mathrm{CE}_{50}>7,20 \mathrm{mg} \cdot \mathrm{L}^{-1}$ \\
\hline Basagran & $3,90 \mathrm{~g} \cdot \mathrm{L}^{-1}$ & $0,96 \mathrm{mg} \cdot \mathrm{L}^{-1}$ & $4.062,5$ & $2,46 \times 10^{-4}$ & $C E_{50}>9,6 \mathrm{mg} \cdot \mathrm{L}^{-1}$ \\
\hline Sírius & $182,11 \mathrm{mg} \cdot \mathrm{L}^{-1}$ & $20 \mu \mathrm{g} \cdot \mathrm{L}^{-1}$ & $9.105,5$ & $0,11 \times 10^{-3}$ & $\mathrm{CE}_{50}>200 \mu \mathrm{g} \cdot \mathrm{L}^{-1}$ \\
\hline Nominee & $1,06 \mathrm{~g} \cdot \mathrm{L}^{-1}$ & $50 \mu \mathrm{g} \cdot \mathrm{L}^{-1}$ & 21.200 & $0,47 \times 10^{-4}$ & $\mathrm{CE}_{50}>500 \mu \mathrm{g} \cdot \mathrm{L}^{-1}$ \\
\hline Ally & $2,10 \mathrm{~g} \cdot \mathrm{L}^{-1}$ & $2 \mu g \cdot L^{-1}$ & 1.050 .000 & $0,09 \times 10^{-5}$ & $\mathrm{CE}_{50}>20 \mu \mathrm{g} \cdot \mathrm{L}^{-1}$ \\
\hline
\end{tabular}

${ }^{1}$ RODRIGUES e ALMEIDA (2005); 2 Índice de segurança = CE50/concentração estimada; ${ }^{3}$ Coeficiente de risco = concentração estimada/CE50; *Com risco de impacto ecológico.

Dependendo da metodologia, o grau de risco ambiental dos agroquímicos pode apresentar variações mesmo com o uso de bioindicadores similares como o zooplâncton. JOST (2003) avaliou o impacto de herbicidas e inseticidas sobre a comunidade zooplanctônica natural em parcelas de arroz irrigado. Seus resultados mostraram efeitos sobre zooplâncton com diminuição na densidade dos organismos, inclusive para produtos que não demonstraram risco de impacto sobre $D$. magna (como quincloraque e pirazossulfurom etílico). É possível que naquele estudo, os herbicidas quincloraque e pirazossulfurom etílico tenham reduzido a densidade de produtores primários (fitoplâncton) (TAMANAHA, 2004), comprometendo a sobrevivivência de organismos herbívoros como o zooplâncton (RESGALLA JÚNIOR et al., 2003; USEPA, 1999).

Os índices de risco apresentados neste trabalho são limitados para possíveis efeitos antagônicos, aditivos ou sinergéticos que podem ocorrer entre misturas de produtos, prática comum nas condições de campo da cultura do arroz irrigado. $\mathrm{Na}$ avaliação da possível interação na aplicação associada de 
quincloraque + pirazossulfurom etílico, os valores de $\mathrm{CE}_{50}$ foram reduzidos pela metade em relação aos valores originais indicando efeitos aditivos entre os produtos (Tabela 3 ). $\mathrm{O}$ uso conjugado de agroquímicos, especialmente de herbicidas e inseticidas, poderia resultar em efeitos sinérgicos sobre a biota aquática.

\title{
QUADRO 3 - VALORES DE CE $_{50}$; 48 HORAS PARA OS TESTES DE TOXICIDADE COM D. magna SOB EFEITO DE FACET E SIRIUS ISOLADOS E EM CONJUNTO
}

\begin{tabular}{|l|c|c|c|}
\hline & $\begin{array}{c}\text { Facet } \\
\text { (isolado) }\end{array}$ & $\begin{array}{c}\text { Sírius } \\
\text { (isolado) }\end{array}$ & $\begin{array}{c}\text { Facet + Sírius } \\
\text { (em conjunto) }\end{array}$ \\
\hline Grupo químico & Quincloraque & Pirazossulfurom & $\begin{array}{c}\text { Quincloraque } \\
\text { Pirazossulfurom }\end{array}$ \\
\hline CE $_{50}$ (i.a.) & $134,31 \mathrm{mg} \cdot \mathrm{L}^{-1}$ & $182,11 \mathrm{mg} \cdot \mathrm{L}^{-1}$ & $\begin{array}{l}60,49 \mathrm{mg} \cdot \mathrm{L}^{-1} \\
75,61 \mathrm{mg} \cdot \mathrm{L}^{-1}\end{array}$ \\
\hline $\begin{array}{l}\text { Intervalo de confiança } \\
\text { (IC 95\%) (i.a.) }\end{array}$ & $\pm 7,9$ & $\pm 8,12$ & $\pm 11,54$ \\
\hline
\end{tabular}

\section{CONCLUSÃO}

Os resultados deste trabalho sugerem que os herbicidas oxadiazona e oxyfluorfem e os inseticidas lambdacialotrina, carbofurano e fipronil apresentam maior potencial de risco de impacto ecológico sobre o bioindicador D. Magma. Os herbicidas 2,4-D, metsulfurom-metílico, carbofentrazona etílica, clomazona, bispiribaque-sódico, quincloraque, pirazossulfurom etílico e bentazona apresentam risco de impacto mínimo a leve sobre o bioindicador $D$. magma.

\begin{abstract}
ACUTE TOXICITY AND RISK ASSESMENT OF HERBICIDES AND INSECTICIDES USED IN PADDY RICE ON CLADÓCERA Daphnia magna

The objective of this research was to determine the $\mathrm{CE}_{50}$; $48 \mathrm{~h}$ of some of the herbicides and insecticides mostly used in paddy rice and to estimate the risks coefficient (deterministic analysis), classifying the chemicals toxicity on Daphnia magna. The following pesticides were evaluated: herbicides - oxyfluorfen, oxadiazon, carfentrazone-ethyl, clomazone, quinclorac, pyrazosulfuron-ethyl, 2,4-D, bispyribac-sodium, metsulfuron-methyl and bentazon; insecticides - lambdacyhalothrin, fipronil and carbofuran. The results showed that herbicides oxyfluorfen, oxadiazon and the insecticides lambdacyhalothrin, fipronil and carbofuran are the chemicals that present the highest toxicity to $D$. magna. The obtained $\mathrm{CE}_{50} 48 \mathrm{~h}$ values in mg. $\mathrm{L}^{-1}$, were $3.18 ; 3.54 ; 12.252 ; 14.35 ; 134.31 ; 182.11 ; 407.3 ; 1,060 ; 2,100 ; 3,900 ; 0.00021 ; 0.15$ and 0.32 , respectively for oxyfluorfen, oxadiazon, carfentrazone-ethyl, clomazone, quinclorac, pyrazosulfuron-ethyl, 2,4-D, bispyribacsodium, metsulfuron-methyl, bentazon, lambdacyhalothrin, fipronil and carbofuran. Considering the chemicals label rate dissolved in a $10 \mathrm{~cm}$ water depth in paddy rice, the herbicides oxyfluorfen, oxadiazon and the insecticides lambdacyhalothrin, fipronil and carbofuran deserve careful irrigation water managing.
\end{abstract}

KEY-WORDS: OXYFLUORFEN; OXADIAZON; CARFENTRAZONE-ETHYL; CLOMAZONE; QUINCLORAC; PYRAZOSULFURON-ETHYL; 2,4-D; BISPYRIBAC-SODIUM; METSULFURON-METHYL; BENTAZON; LAMBDACYHALOTHRIN; FIPRONIL; CARBOFURAN; ECOTOXICOLOGY. 


\section{REFERÊNCIAS}

1 ABNT. Associação Brasileira de Normas Técnicas. NBR 12713: ecotoxicologia aquática - toxicidade aguda - método de ensaio com Daphnia spp (Cladocera, Crustacea). Rio de Janeiro, 2004.

2 CONAB. Companhia Nacional de Abastecimento. Estimativa de safras: 2005/2006. Disponível em: www.conab.gov.br. Acesso em: outubro, 2006.

3 EPAGRI. Empresa de Pesquisa Agropecuária e Extensão Rural de Santa Catarina. Sistema de produção de arroz irrigado em Santa Catarina (pré-geminado). 2. ed. rev. e atual. Florianópolis, 2005. 87 p. (Epagri. Sistema de Produção, 32)

4 FRELLO, C.P. Avaliação da toxicidade aguda do pesticida carbofuran utilizando reativos biológicos Poecilia reticulata e Daphnia magna. Florianópolis, 1998. 96 p. Dissertação (Mestrado em Engenharia Ambiental), Universidade Federal de Santa Catarina.

5 HELFRICH, L.A.; WEIGMANN, D.L.; HIPKINS, P.; STINSON, E.R. Pesticides and aquatic animals: a guide to reducing impacts on aquatic systems. 1996. Disponível em: www.ext.vt.edu/pubs/waterquality/ 420-013/420-013.pdf. Acesso em: abril, 2003.

6 JONSSON, C.M.; MAIA, A.H.N. Toxicidade aguda do herbicida clomazone no peixe Hyphessobrycon scholzei: avaliação da concentração letal mediana e de alterações no conteúdo de nutrientes. Pesticidas: revista de ecotoxicologia e meio ambiente, Curitiba, v. 8, p.101-110, 1998.

7 JOST, G.F. Avaliação dos impactos de agroquímicos sobre a comunidade zooplanctônica da água de irrigação do arroz, utilizando Copépodas (Crustacea) como bioindicadores. Itajaí, 2003. 63 p. Monografia (Bacharel em Biologia), Universidade do Vale do Itajaí.

8 KOKTA, C.; ROTHERT, H.A. Hazard and risk assessment scheme for evaluating effects on earthworms: the approach in the Federal Republic of Germany. In: GREIG-SMITH, P.W.; BECKER, H.; EDWARDS, P.J.; HEIMBACH, F. (eds.). Ecotoxicology of earthworm. Andover: Intercept, 1992. p.169-176.

9 RESGALLA JÚNIOR, C.; NOLDIN, J.A.; SANTOS, A.L.; SATO, G.; EBERHARDT, D.S. Toxicidade aguda de herbicidas e inseticida utilizados na cultura de arroz irrigado sobre juvenis de carpa (Cyprinus carpio). Pesticidas: revista de ecotoxicologia e meio ambiente, Curitiba, v. 12, p. 59-68, 2002.

10 RESGALLAJÚNIOR, C.; NOLDIN, A.J.; TAMANAHA, M.S.; DESCHAMPS, F.C.; EBERHARDT, S.D.; SILVEIRA, R.M.; MÁXIMO, M.V.; LAITANO, K.S.; JOST, G.F.; RORIG, L.M. Testes de toxicidade e análise de risco de agroquímicos In: CONGRESSO BRASILEIRO DE ARROZ IRRIGADO, 3., e REUNIÃO DA CULTURA DO ARROZ IRRIGADO, 25., 2003, Balneário Camboriú-SC. Anais... Itajaí: Epagri. 2003. p.798-807.

11 RODRIGUES, B.N.; ALMEIDA, F.S. Guia de herbicidas. 5. ed. Londrina: Ed. dos Autores, 2005. 592 p.

12 SEUS, L.G. Toxicidade aguda e crônica em alevinos de jundiá ( $R$ hamdia sp.) submetidos aos principais herbicidas utilizados na lavoura de arroz irrigado no RS. Pelotas, 2002. 73 p. Dissertação (Mestrado em Zootecnia de Produção), Universidade Federal de Pelotas.

13 SOLOMON, K.R. Advances in the evaluation of the toxicological risks of herbicides to the environment. In: CONGRESSO BRASILEIRO DA CIÊNCIA DAS PLANTAS DANINHAS, 21., Caxambu, MG. Anais ... Caxambu: SBCPD, 1997. p.163-172.

14 SOSBAI. Sociedade Sul-Brasileira de Arroz Irrigado. Arroz irrigado: recomendações técnicas da pesquisa para o Sul do Brasil. Santa Maria, 2005. 159 p.

15 TAMANAHA, M.S. Estrutura das assembléias fitoplanctônicas e testes de inibição de crescimento algal: avaliação dos efeitos dos herbicidas e inseticidas utilizados na cultura do arroz irrigado na Região de Itajaí, SC. Itajaí, 2004. 98 p. Dissertação (Mestrado em Ciência e Tecnologia Ambiental), Universidade do Vale do Itajaí.

16 USEPA. Environmental Protection Agency. ECOFRAM aquatic report. 1999. Disponível em: http:// www.epa.gov. Acesso em: dezembro, 2003.

17 USEPA. Environmental Protection Agency. Short-term methods for estimating the chronic toxicity of effluents and receiving waters of freshwater organisms. $4^{\text {th }}$ ed. Washington, 2002. (EPA-821-R-02-013). 
18 ZAGATTO, P.A.; GOLDSTEIN, E.G. Estudo comparativo entre as taxas de reprodução de Daphnia similis Claus, 1876 e Daphnia magna Straus, 1820: resultados preliminares. In: SIMPÓSIO BRASILEIRO DE AQUICULTURA, 3., 1984, São Carlos: Anais... São Carlos: UFSCar /CESP, 1984. p.411-424.

\section{AGRADECIMENTOS}

Os autores agradecem à Embrapa/Fundagro/Prodetab, conv. 1020003/0056-6, pelo apoio financeiro e administrativo, o qual viabilizou a realização deste trabalho de pesquisa. 